Supporting Information

\title{
Direct Conversion of Sugarcane Bagasse into Injection-Moldable Cellulose-Based Thermoplastic via Homogeneous Esterification with Mixed Acyl Groups
}

\author{
Shiori Suzuki, ${ }^{\ddagger}$ Hibiki Hikita, Stephanie C. Hernandez, Naoki Wada, and Kenji Takahashi ${ }^{*}$ \\ Faculty of Natural System, Institute of Science and Engineering, Kanazawa University, \\ Kakuma-machi, Kanazawa, Ishikawa 920-1192, Japan. \\ *Corresponding author e-mail: ktkenji@staff.kanazawa-u.ac.jp \\ *Present affliation: Department of Biomaterial Science, Graduate School of Agricultural and Life Sciences, \\ The University of Tokyo, 1-1-1, Yayoi, Bunkyo-ku, Tokyo 113-8657, Japan
}

\section{Table of Contents}

DS Calculation for Polysaccharide Derivatives

Page \#

Peracylation of Commercial Cellulose Acetate (CA)

Optimization of Long Acyl Chain Structure for Improving Thermal Processability of CA Derivative

Identification of LAD synthesized from bagasse by FT-IR and ${ }^{1} \mathrm{H} \&{ }^{13} \mathrm{C}$ NMR

Identification of CADs from Avicel and Cellulose Pulps by FT-IR and ${ }^{1} \mathrm{H}$ NMR

DS of De/Ac groups and Molecular Weight Distributions of CADs

Characterization of LAD synthesized from bagasse by TGA, DSC, flow-tester, and GPC

TG, DSC, and Heat-flow Curves of CADs

Pictures of the Injection-molded Specimen of CADs.

References

$\mathrm{S} 10$

$\mathrm{S} 10$ 


\section{DS Calculation for Polysaccharide Derivatives.}

According to our previous reports, residual $\mathrm{OH}$ groups in polysaccharide derivatives were perbenzoylated, and the DS values were estimated from ${ }^{1} \mathrm{H}$ NMR spectra of the perbenzoylated products.

The polysaccharide derivatives $(100 \mathrm{mg})$ was dissolved into chloroform $(4-10 \mathrm{~mL})$ and triethylamine $(0.44 \mu \mathrm{L}, 3.1$ $\mathrm{mmol})$ at room temperature for more than $1 \mathrm{~h}$. Benzoyl chloride $(0.36 \mu \mathrm{L}, 3.1 \mathrm{mmol})$ was added and stirred at room temperature for $24 \mathrm{~h}$. The reaction mixture was then poured into methanol $(300 \mathrm{~mL})$. The precipitate was collected by filtration and washed with methanol, repeatedly. Subsequently, the gel-like solid was dissolved in chloroform (20-30 $\mathrm{mL}$ ) with stirring at room temperature for more than $1 \mathrm{~h}$. The resulting solution was concentrated by a rotary evaporator at $40{ }^{\circ} \mathrm{C}$ and reprecipitated into methanol $(300 \mathrm{~mL})$. The residue was washed with methanol and dried under vacuum at $70{ }^{\circ} \mathrm{C}$ for $24 \mathrm{~h}$. The ${ }^{1} \mathrm{H}$ NMR of the benzoylated polysaccharide derivative was measured in DMSO- $d_{6}$ containing 0.03 vol\% TMS. In addition, in order to determine the molar ratio between long and short acyl groups, ${ }^{1} \mathrm{H}$ NMR of the polysaccharide derivative (without perbenzoylation) was measured in chloroform- $d_{1}$.

The molar ratio between long group (e.g., De group) and short acyl groups (i.e., Ac group) and that between long acyl group and residual $\mathrm{OH}$ group (i.e., benzoyl group) were calculated by formula (1) and (2), respectively.

Long acyl group : Short acyl group $(\mathrm{mol} / \mathrm{mol})=\frac{I_{\delta 1.0-2.5}-N}{3}: \frac{I_{\delta 0.8-1.0}}{3}$

OH group : Long acyl group $(\mathrm{mol} / \mathrm{mol})=\frac{I_{\delta 6.2-8.4}}{5}: \frac{I_{\delta 0.7-0.9}}{3}$

where $I_{\delta 1.0-2.5}$ denotes the integrals of the multiple peaks of Ac and long acyl groups except for the end methyl group, and $I_{\delta 0.8-1.0}$ and $I_{\delta 0.7-0.9}$ denotes that of the end methyl group while the integral areas slightly differ because of the solvent effect of DMSO- $d_{6}$ and chloroform- $d_{1}$. The value of $N$ follows as the number of protons contained in the methylene groups of the long acyl chain minus that of the end methyl group $=3$. Thus, when the long acyl group was De groups, the $N$ value would be $16 . I_{\delta 6.2-8.4}$ denotes the integral of peaks assigned to the ring protons in benzoyl group.

Anhydrous glucose unit (AGU) in cellulose has three $\mathrm{OH}$ groups, while anhydrous xylose unit (AXU) in xylan has two OH groups. Therefore, the OH conversion and DS were calculated by formula (3) and (4), respectively.

$\mathrm{OH}$ conv. (\%) $=\frac{R_{\text {Long }}+R_{\text {Short }}}{R_{\text {Long }}+R_{\text {Short }}+R_{\text {OH }}} \times 100$

DS $(-)=\mathrm{OH}$ conv. $(\%) \div 100 \times$ Conversion factor

Where $\mathrm{R}$ denotes the molar ratio calculated by formula (1) and (2) and conversion factors are 3 for cellulose derivative and 2 for xylan derivative. 


\section{Peracylation of Commercial Cellulose Acetate (CA).}

A commercial CA (DS $\left.\mathrm{Ac}: 2.49, \mathrm{M}_{\mathrm{w}}: 10^{5} \mathrm{gmol}^{-1}\right)$ of ACROS ORGANICs, a Thermo Fisher Scientific brand, was subjected to peracylation using acyl halides with different numbers of carbon $(\mathrm{C}=4-18)$ and triethylamine as both a catalyst and $\mathrm{HCl}$ scavenger. $\mathrm{CA}(5.0 \mathrm{~g},[\mathrm{OH}]=63 \mathrm{mmol})$ was dissolved in dichloromethane $(100 \mathrm{~mL})$ at room temperature, and triethylamine $(12.9 \mathrm{~mL}, 1.5 \mathrm{eq} . /[\mathrm{OH}])$ was added to the homogeneous solution. Then, an equimolar amount of acyl halide (1.5 eq./[OH]) with the triethylamine was dropped with cooling in an ice bath $\left(\sim 0{ }^{\circ} \mathrm{C}\right)$ and reacted at room temperature by stirring for $22 \mathrm{~h}$. The resultant solution was precipitated in methanol $(800 \mathrm{~mL})$, and the precipitate was filtered and washed with methanol, repeatedly. The pale-yellow residue was dissolved in acetone (100 $\mathrm{mL}$ ), and the resultant solution was filtered to remove impurities such as triethylamine acetate. The filtrate was poured into methanol $(800 \mathrm{~mL})$, and the precipitate was collected by filtration. After vacuum-drying at $50{ }^{\circ} \mathrm{C}$, the peracylated CA derivatives were obtained. The yields and DS of the CA derivatives are listed in Table S1.

Table S1 Yields and DS of CA derivatives synthesized by peracylation of a commercial CA

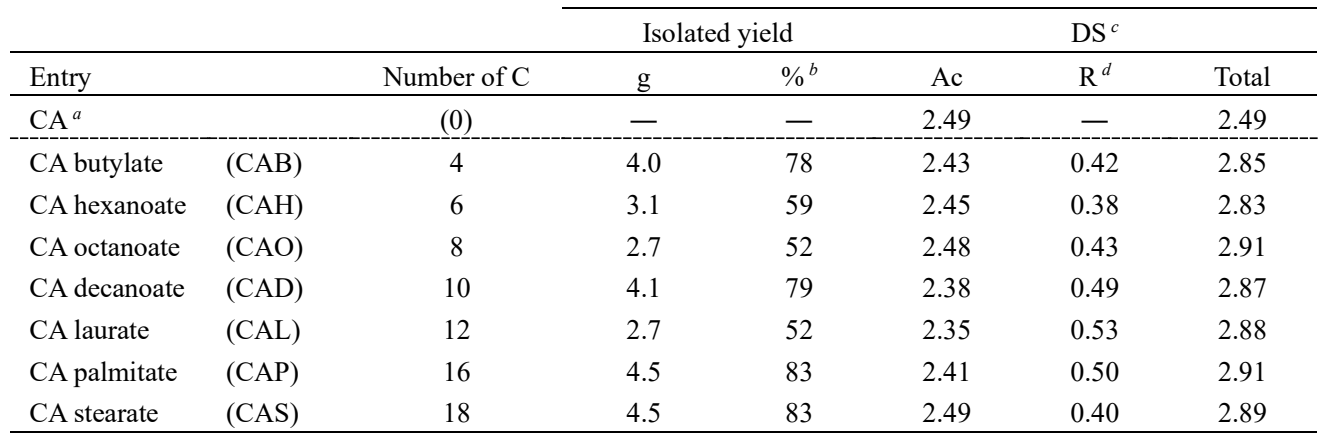

${ }^{a}$ Starting material. ${ }^{b}$ Calculated by a theoretical yield of each product, hypothesized as all residual OH groups in CA were substituted by acyl halides. ${ }^{c}$ Determined by ${ }^{1} \mathrm{H}$ NMR of $\mathrm{CAP}$ in $\mathrm{CDCl}_{3}$ and the per-benzoylated derivative in DMSO-d $6 .{ }^{d} \mathrm{DS}$ of the corresponded acyl groups. 
Optimization of Long Acyl Chain Structure for Improving Thermal Processability of CA Derivative.

Tanaka et al. demonstrated that a mixed substitution of long/short acyl chains improved the thermal processability (i.e., heat-melting and degradation temperatures) of cellulose ester due to the internal plasticization effect of the long acyl chain, and lower DS of the long acyl groups (e.g., $\mathrm{DS}_{\text {long }}=0.4, \mathrm{DS}_{\text {short }}=2.6$ ) resulted in higher mechanical property. ${ }^{1}$

Then, in order to determine the best number of carbon of the long acyl group for improving the thermal processability of the short-chain-rich CA derivatives, a commercial CA $\left(\mathrm{DS}_{\mathrm{Ac}}=2.49\right)$ was per- acylated using acyl halides of different acyl chain lengths $(C=4-18)$. The thermal properties of the resultant derivatives were analyzed by TGA and DSC. Since all the CA derivatives did not show a melting point in DSC curves, and then, the thermal processability was estimated from a difference between $T_{\mathrm{d}-10 \%}$ and $T_{\mathrm{g}}$.

As shown in Figure S1, as the carbon number of the acyl chain groups increased from 4 to $10, T_{\mathrm{d}-10 \%}$ of the CA derivatives kept at approximately $350{ }^{\circ} \mathrm{C}$ and higher than that of the original $\mathrm{CA}\left(328^{\circ} \mathrm{C}\right)$. On the other hand, $T_{\mathrm{g}}$ decreased from 176 to $123{ }^{\circ} \mathrm{C}$ due to increased spacing between cellulose chains and dilution of secondary bonding, increasing the mobility of the main chains (i.e., plasticizer effect). However, as the number of carbon in the acyl chain length became larger than $10, T_{\mathrm{g}}$ began to increase slightly, as steric hindrance of neighboring bulky substituents restricts rotation about the glycosidic ether linkages of the cellulose backbone, stiffening the chain. These characteristic behavior of changing $T_{\mathrm{g}}$ of cellulose derivatives with long acyl chain structures is almost constant with the previously reported results. ${ }^{1,2}$ Under the examined conditions in this study, CAD $(\mathrm{C}=10)$ showed the lowest $T_{\mathrm{g}}\left(123{ }^{\circ} \mathrm{C}\right)$ with sufficient high $T_{\mathrm{d}-10 \%}\left(346^{\circ} \mathrm{C}\right)$, suggesting that the substitution with a small amount of decanoyl (De) group into CA would be most effective to improve the actual thermal processability, for example, toward injection molding. 
Identification of LAD synthesized from bagasse by FT-IR and ${ }^{1} \mathrm{H} \&{ }^{13} \mathrm{C} N M R$.

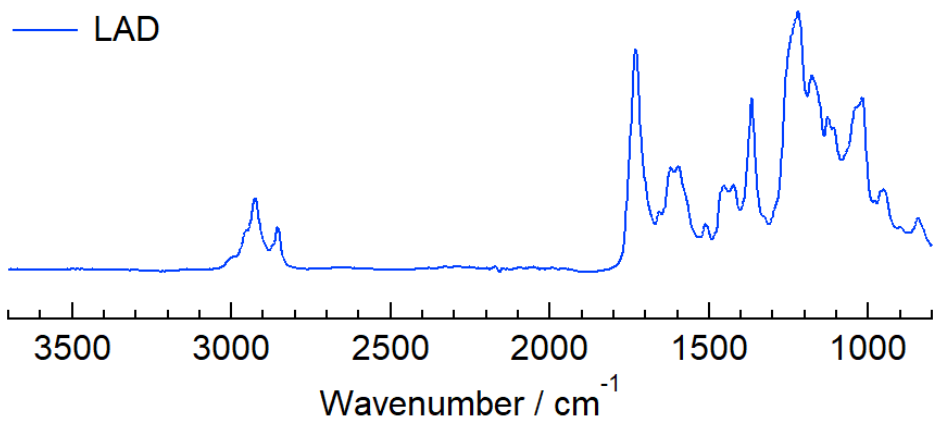

Figure S2. ATR-mode FT-IR spectra of LAD synthesized from bagasse.

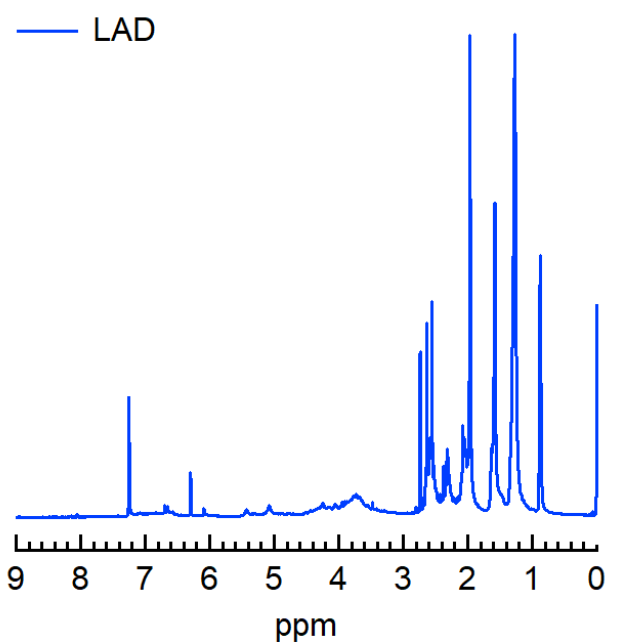

Figure S3. ${ }^{1} \mathrm{H}$ NMR spectra of LAD synthesized from bagasse measured in chloroform- $d_{1}$ at $55^{\circ} \mathrm{C}$.
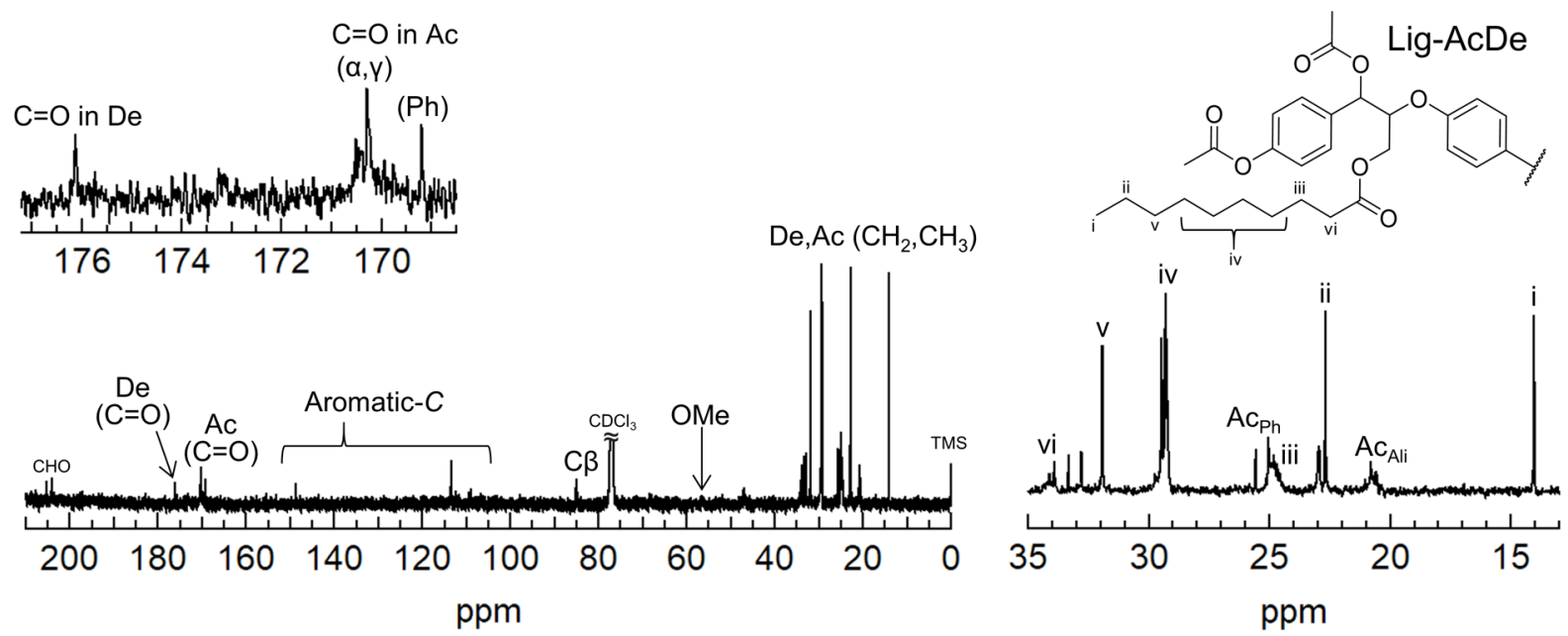

Figure S4. ${ }^{13} \mathrm{C}$ NMR spectra of LAD synthesized from bagasse measured in chloroform- $d_{1}$ at $55{ }^{\circ} \mathrm{C}$. 
Identification of CADs from Cellulose Pulps by FT-IR and ${ }^{1} \mathrm{H}$ NMR.

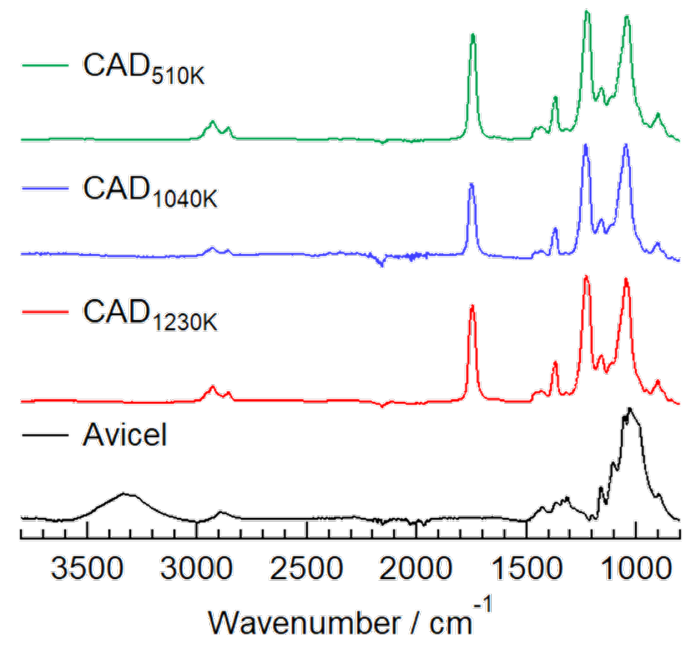

Figure S5. ATR-mode FT-IR spectra of CADs synthesized from cellulose pulps.

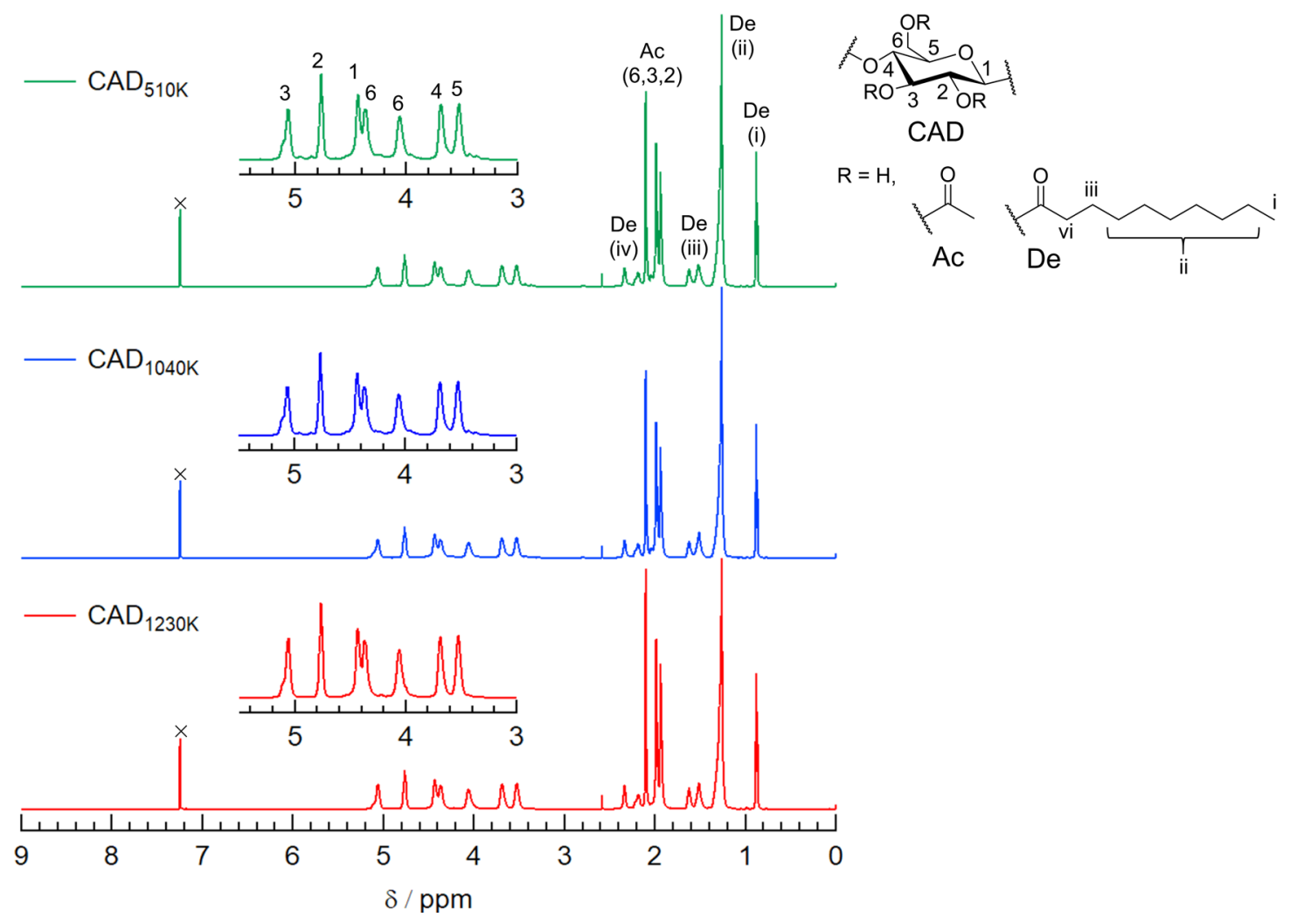

Figure S6. ${ }^{1} \mathrm{H}$ NMR spectra of CADs synthesized from cellulose pulps measured in chloroform- $d_{1}$ at $55^{\circ} \mathrm{C}$. 


\section{DS of De/Ac groups and Molecular Weight Distributions of CADs.}

The introduction of De and Ac groups in the targeted DS ( $\mathrm{De}: \mathrm{Ac}=0.5: 2.5$ ) into the cellulosic pulps was achieved by one-pot two-step transesterification in EmimOAc with adding VDe and IPAc, in order. Although the molar ratio of De group slightly decreased from $19 \%$ to $16 \%$ with increasing the molecular weights of the original cellulose, the DS of De and Ac groups in all CADs were successfully controlled as to be 0.5 and 2.5, respectively, by the one-pot two-step homogeneous reaction.

Table S2 DS of De/Ac groups and molecular weight distribution of CADs

\begin{tabular}{lccccc} 
& \multicolumn{2}{c}{ Molar ratio (DS) } & \multicolumn{2}{c}{$\%(-)$} & \multicolumn{2}{c}{ Molecular weight distribution $^{b}$} \\
\hline Product & De & Ac & $M_{\mathrm{W}} / 10^{4} \mathrm{~g} \mathrm{~mol}^{-1}$ & $\bigoplus^{c}$ & $\mathrm{DP}_{\mathrm{w}}{ }^{d}$ \\
\hline $\mathrm{CAD}_{510 \mathrm{~K}}$ & $17(0.5)$ & $82(2.5)$ & 51 & 3.3 & 1472 \\
$\mathrm{CAD}_{1040 \mathrm{~K}}$ & $17(0.5)$ & $81(2.4)$ & 104 & 4.0 & 3022 \\
$\mathrm{CAD}_{1230 \mathrm{~K}}$ & $16(0.5)$ & $83(2.5)$ & 123 & 2.8 & 3572 \\
\hline
\end{tabular}

a Determined by ${ }^{1} \mathrm{H}$ NMR of the $\mathrm{CADs}$ in $\mathrm{CDCl}_{3}$ and the perbenzoylated derivatives in DMSO- $\mathrm{d}_{6} \cdot{ }^{b}$ Estimated by SEC using chloroform as eluent and polystyrene standards. ${ }^{c} M_{\mathrm{w}} / M_{\mathrm{n}} \cdot{ }^{d} M_{\mathrm{w}} / 344$.

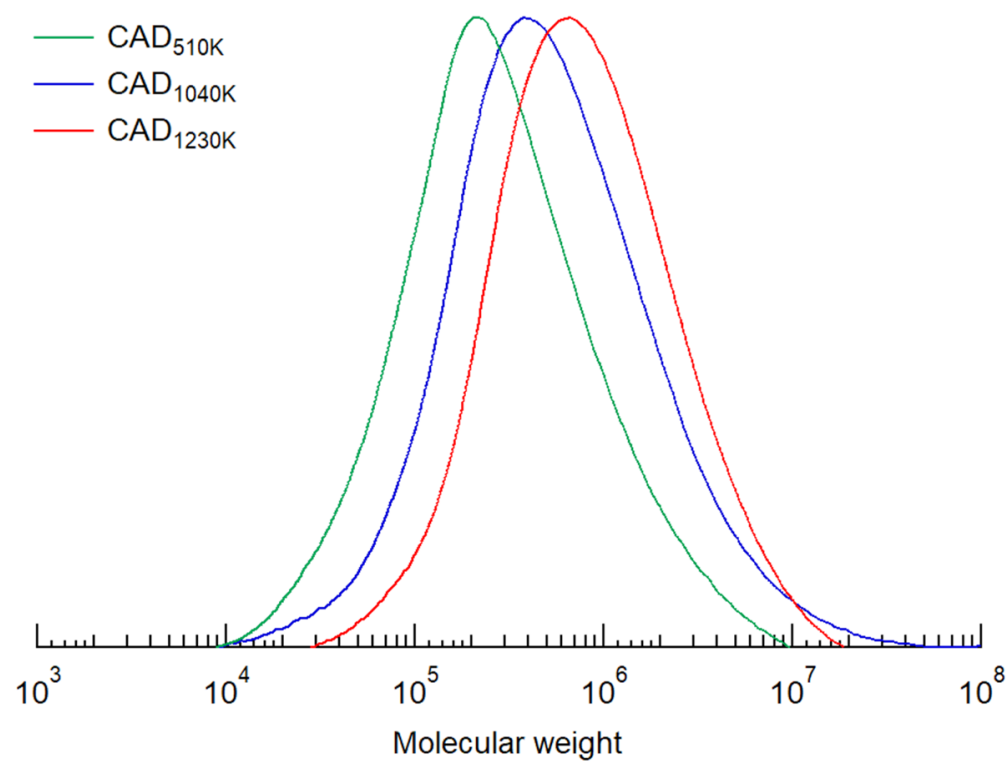

Figure S7. Molecular distributions of CADs synthesized from cellulose pulps, measured by SEC in chloroform as an eluent with polystyrene standards. 
Characterization of LAD synthesized from bagasse by TGA, DSC, flow-tester, and GPC.

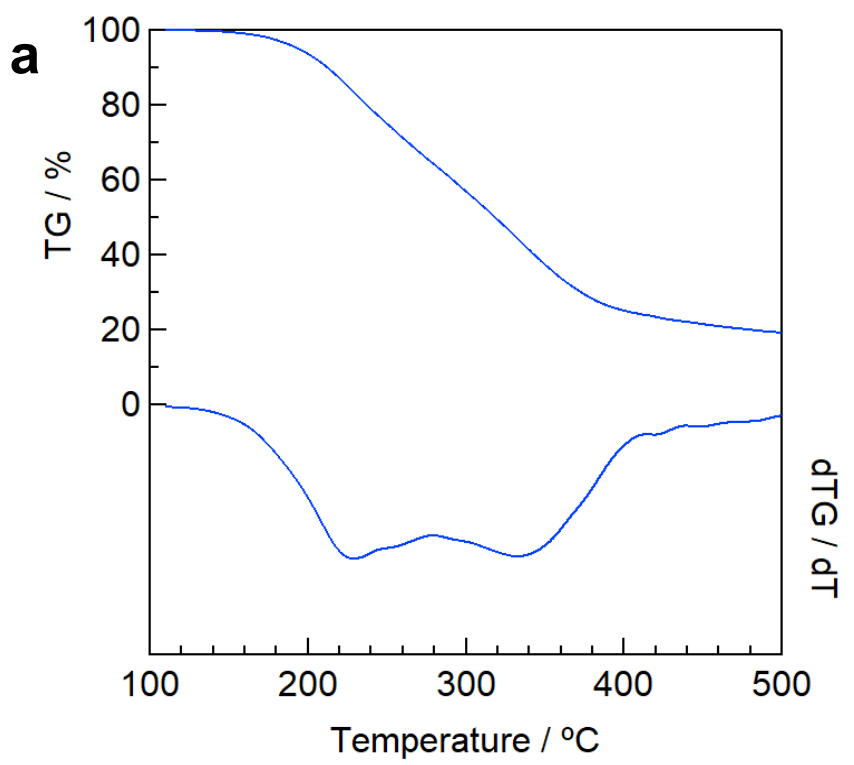

b)

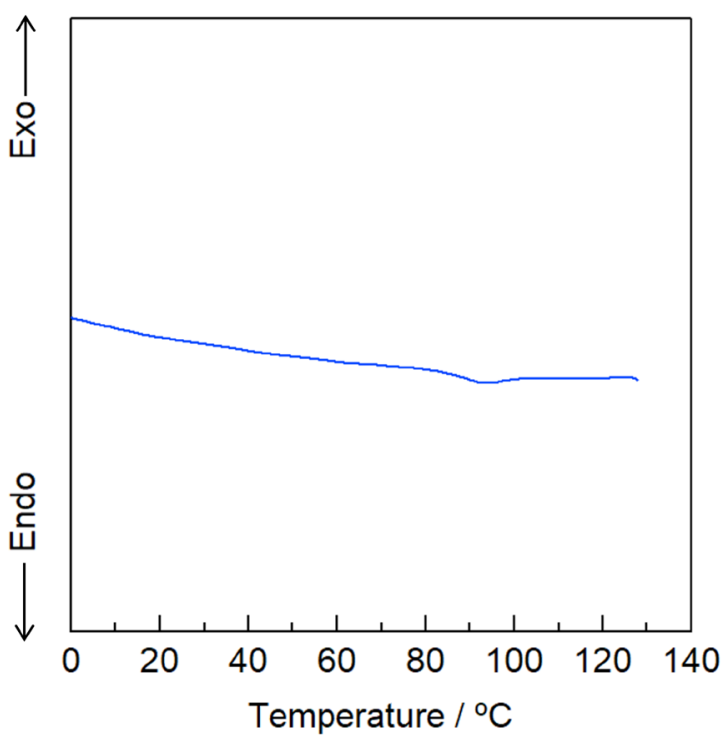

d)

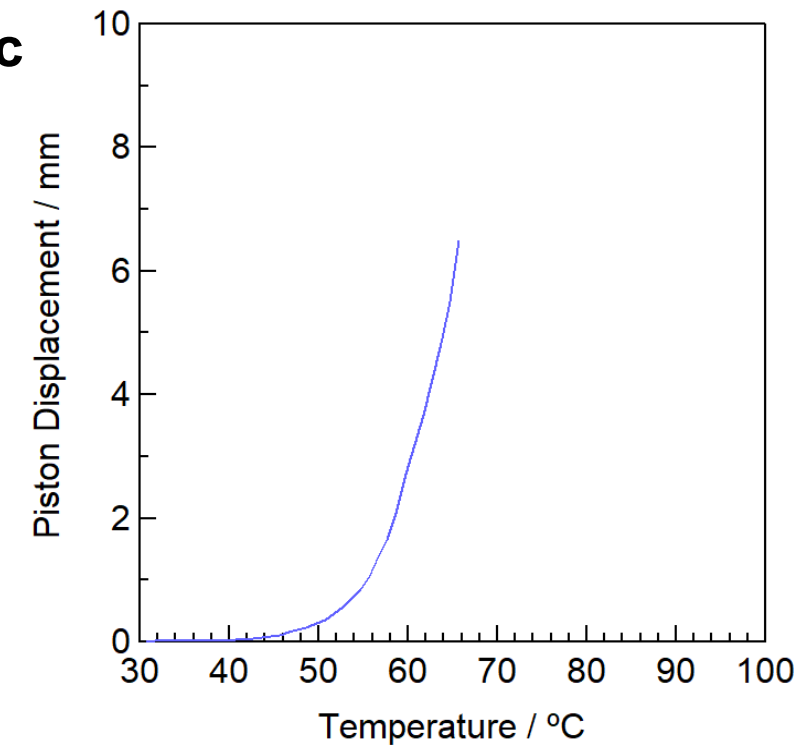

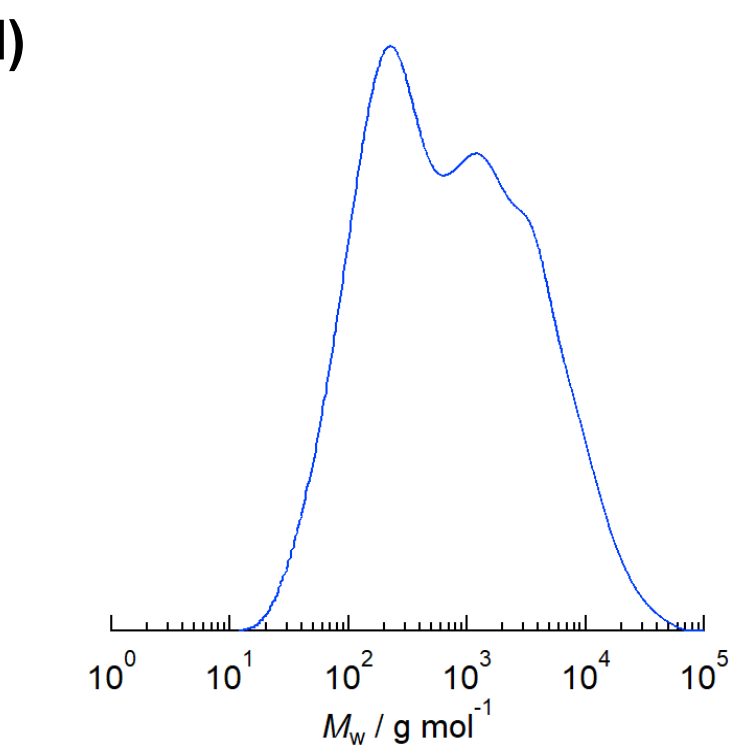

Figure S8. (a) TG and DTG (dTG/dT), (b) DSC ( $2^{\text {nd }}$ heating), and (c) thermal flow curves, and d) molecular weight distributions of LAD synthesized from bagasse. 


\section{TG, DSC, and Heat-flow Curves of CADs.}

Thermal properties of CADs were determined by TGA, DSC, and heat-flow test. With increasing the molecular weight of CADs, the 5\%-weight-loss-temperatures $\left(T_{\mathrm{d}-5 \%}\right)$ slightly increased from 330 to $349{ }^{\circ} \mathrm{C}$ (Figure S9), which are typical of cellulose ester substituted with long acyl chain groups. ${ }^{1,3,4}$ On the other hand, it was found that the molecular weight hardly affected the $T_{\mathrm{g}}$ of CAD. The $T_{\mathrm{g}}$ around $120{ }^{\circ} \mathrm{C}$ was lower than that of similar long/short chain mixed cellulose esters $\left(\sim 140^{\circ} \mathrm{C}\right),{ }^{1,5}$ No endothermic peak was observed in the DSC curves (Figure S10), indicating all CADs were amorphous polymers. These results from DSC analysis suggest that the limited introduction of De groups (DS, $c a$. 0.5 ) successfully prevented the strong interaction between main chain of $\mathrm{CA}^{6,7}$ without crystallization of the long acyl chain moieties. Furthermore, the heat-melting properties such as $T_{\text {flow }}$ and $T_{\text {offset }}$ significantly depended on the molecular weight of CADs (Figure S11). The $T_{\text {offset }}$ increased from $218^{\circ} \mathrm{C}$ to $270{ }^{\circ} \mathrm{C}$ as the molecular weight of CADs increased. This phenomenon is caused by the increase in entanglement between the molecular chains, resulting higher viscosity during heat-melting.

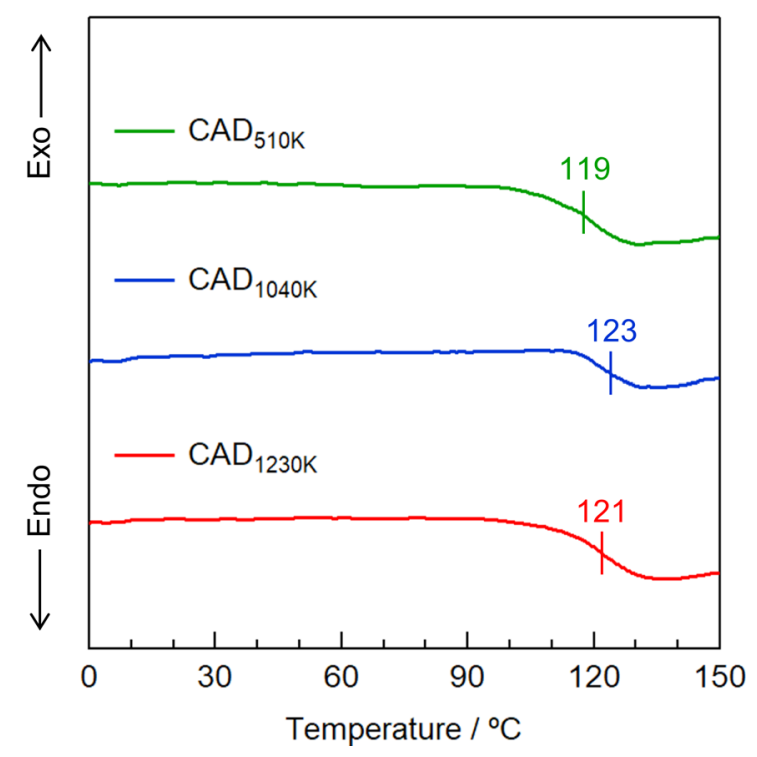

Figure S10. DSC curves $\left(2^{\text {nd }}\right.$ heating) of CADs synthesized from cellulose pulps, measured under $\mathrm{N}_{2}$ atmosphere.

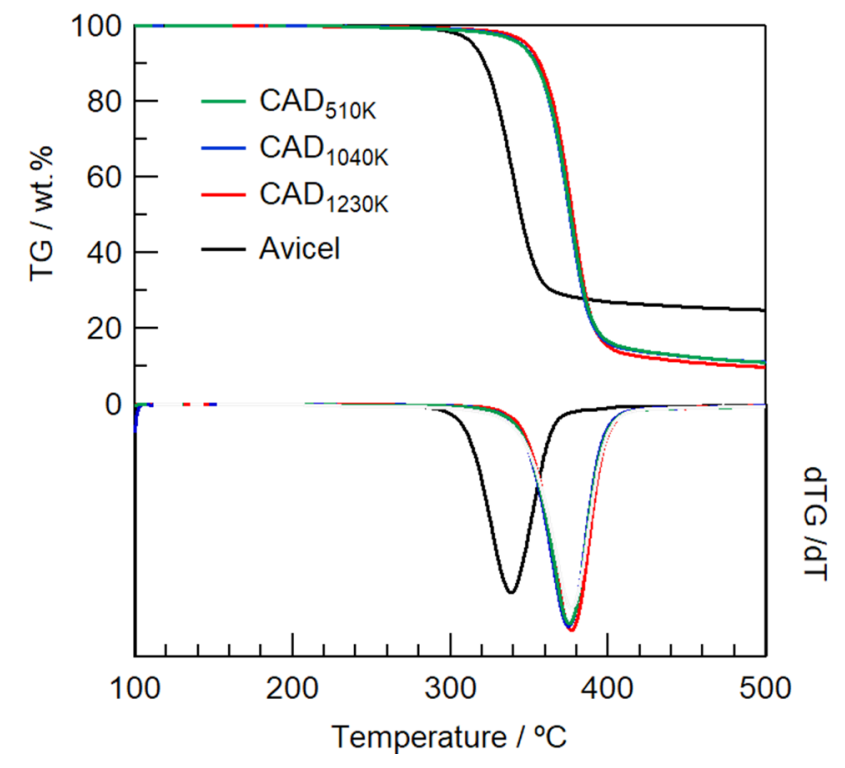

Figure S9. TG curves of CADs synthesized from cellulose pulps, measured under $\mathrm{N}_{2}$ atmosphere.

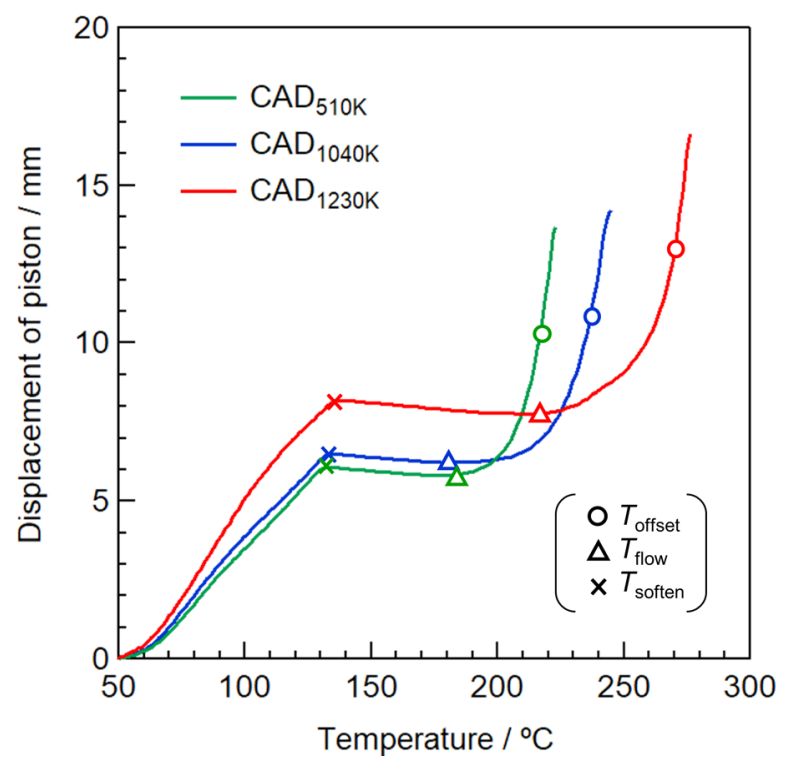

Figure S11. Heat-flow curves of CADs synthesized from cellulose pulps, measured at heating rate of $2{ }^{\circ} \mathrm{C} \mathrm{min}^{-1}$. 


\section{Pictures of the Injection-molded Specimen of CADs.}

\begin{tabular}{|l|l|l|l|}
\hline & $\mathrm{CAD}_{510 \mathrm{~K}}$ & $\mathrm{CAD}_{1040 \mathrm{~K}}$ & $\mathrm{CAD}_{1230 \mathrm{~K}}$ \\
\hline $\begin{array}{l}\text { Dumbbell-type } \\
\text { specimen }\end{array}$ & & & \\
\hline $\begin{array}{l}\text { Strip-type } \\
\text { specimen }\end{array}$ & & & \\
\hline
\end{tabular}

Figure S12. Pictures of the injection-molded specimen of CADs synthesized from cellulose pulps with different molecular weights for tensile and bending tests.

\section{References}

1. S. Tanaka, T. Iwata and M. Iji, ACS Sustainable Chemistry \& Engineering, 2017, 5, 1485-1493.

2. J. E. Sealey, G. Samaranayake, J. G. Todd and W. G. Glasser, Journal of Polymer Science Part B: Polymer Physics, 1996, 34, 1613-1620.

3. W. G. Glasser, G. Samaranayake, M. Dumay and V. Davé, Journal of Polymer Science Part B: Polymer Physics, 1995, 33, 2045-2054.

4. X. Cao, X. Peng, L. Zhong, S. Sun, D. Yang, X. Zhang and R. Sun, Cellulose, 2014, 21, 581-594.

5. $\quad$ M. Iji, S. Moon and S. Tanaka, Polymer Journal, 2011, 43, 738-741.

6. H. M. Shaikh, K. V. Pandare, G. Nair and A. J. Varma, Carbohydrate Polymers, 2009, 76, 23-29.

7. K. Peredo, H. Reyes, D. Escobar, J. Vega-Lara, A. Berg and M. Pereira, Carbohydrate Polymers, 2015, 117, 1014-1020. 